\title{
On the production of multicharge ions in planetary nebulae
}

\author{
Subhon Ibadov ${ }^{1,2}$ \\ ${ }^{1}$ Institute of Astrophysics, Tajik Academy of Sciences, Dushanbe, Tajikistan \\ ${ }^{2}$ Sternberg Astronomical Institute, Moscow State University, Moscow, Russia \\ email: ibadovsu@yandex.ru
}

\begin{abstract}
Production of multicharge ions due to generation of hot expanding plasma blobs, short-living "compound particles", at high-velocity collisions between dust grains of a planetary nebula and dust particles of the interstellar medium is analytically considered. Dependence of the multiplicity of charge of produced ions on the relative velocity of colliding dust particles is determined.
\end{abstract}

Keywords. planetary nebulae: general, dust, plasmas, atomic processes

\section{Introduction}

Planetary nebulae ( $\mathrm{PNe}$ ) have high dust content and radiate strongly in the infrared (see, e.g., Kwok et al. 2006). Expansion velocities of PNe cover a wide range including the band $50-100 \mathrm{~km} / \mathrm{s}$, PNe plasma consists of multicharge ions like He II, C III, C IV, O III, O IV, O V, Ne IV, N III, N V, etc. The number of chemical elements in PNe revealed by observations exceeds 20 including ions of metals like Na, Si, Fe, etc. The typical kinetic temperatures of PNe electrons are of the order of $10^{4} \mathrm{~K}$, that is essentially less than the ionization potentials of atoms. It means that the ionization of atoms of a surrounding gaseous nebula will be produced by ultraviolet (UV) photons emitted by a central star (Aller 1984; Kaler 1976; Kostyakova 1982; Kostyakova \& Arkhipova 2009; Kwok 2000).

We will consider possibility of production of multicharge ions in PNe due to generation of hot plasma blobs by high-velocity collisions $(V>30 \mathrm{~km} / \mathrm{s})$ between PNe dust particles and dust particles of the interstellar medium (ISM).

\section{Production of multicharge ions in $\mathrm{PNe}$ by collisions of dust particles}

The specific energy at high-velocity collisions between PNe dust particles and dust particles of the ISM, being more than $10^{13} \mathrm{erg} / \mathrm{g}$, essentially exceeds the one for ionization of neutral atoms. The situation indicates to the possibility of production of multicharge ions due to generation of short-living high-temperature plasma blobs, "compound particles", via such collisions.

Indeed, the initial temperature of the plasma blob $T=T_{*}\left(V / V_{*}\right)^{2}$ with $T=3 \times 10^{5} \mathrm{~K}$ at $V=V_{*}=70 \mathrm{~km} / \mathrm{s}=7 \times 10^{6} \mathrm{~cm} / \mathrm{s}$ (Ibadov 1984, 1985, 1986).

As is known (Zeldovich \& Raizer 1966), the mean most probable multiplicity of ionization in a plasma, $\bar{z}\left(T, n_{e}\right)=z$, is connected with the corresponding ionization potential of ions $I_{z}$ by the transcendental equation

$$
I_{z}=k T \ln \left[\left(A_{1} T^{3 / 2}\right) /\left(z n_{z}\right)\right],
$$


where $k=8.62 \times 10^{-5} \mathrm{eV} \mathrm{K}^{-1}, A_{1}=2\left(2 \pi m k / h^{2}\right)^{3 / 2}=4.8 \times 10^{15} \mathrm{~cm}^{-3} \mathrm{~K}=6 \times$ $10^{21} \mathrm{~cm}^{-3} \mathrm{eV}^{-3 / 2}, n_{z}$ is the number density of $z$-ions, $I_{z}$ is the ionization potential of $z$-ions in $\mathrm{eV}$.

We will present known numerical data on the ionization potentials of atoms analytically, $I_{z}=I(z)$, in the form

$$
I_{z}=I_{1} z^{s_{i}}
$$

where $I_{1}$ is the ionization potential of the neutral atom, so that $z=1$ corresponds to single ionization; $s_{i}=s_{i}(z)$ is the parameter of the curve of ionization potentials: $1<s_{i} \leqslant 2$ and quasi-monotonically rising with increasing $z$ for each sort of atoms.

According to known tables for ionization potentials of atoms (see, e.g., Allen 1977), for Fe atoms we have $s_{i}=1.4$ for $1 \leqslant z \leqslant 8$ and $s_{i}=1.5$ for $9 \leqslant z \leqslant 16$. For oxygen atoms $s_{i}=1.3$ at $1 \leqslant z \leqslant 6$.

Using Eqs. (2.1), (2.2) the dependence of the multiplicity of charge of ions on the relative velocity of colliding dust particles may be found, namely

$$
z=\left(k f_{*} T_{*} / I_{1}\right)^{1 / s_{i}}\left(V / V_{*}\right)^{\left(2+s_{v}\right) / s_{i}},
$$

where $f_{*} \approx 2, s_{v} \approx 0.5$ (Ibadov 1992, 1996).

Eq. (2.3) for oxygen atoms with $I_{1}(\mathrm{O})=13.6 \mathrm{eV}$ and $V=100 \mathrm{~km} / \mathrm{s}$ gives $z=4$.

\section{Conclusions}

Multicharge ions in PNe may be produced not only due to photoionization of gases by UV photons from a central star but also due to high-velocity collisions between dust particles of PNe and ISM.

\section{Acknowledgements}

The author is grateful to the IAUS 283 LOC/SOC for an invitation. The hospitality of the DIC MSU as well as help of Drs. V. P. Arkhipova and G.M. Rudnitskij, SAI MSU, are acknowledged.

\section{References}

Allen, C. W. 1977, Astrophysical Quantities, Mir, Moscow, 446

Aller, L. H. 1984, Physics of Thermal Gaseous Nebulae, D. Reidel, Dordrecht, 350

Ibadov, S. 1984, Astron. Circ. (USSR) 1353, 1

Ibadov, S. 1985, Astron. Circ. (USSR) 1384, 1

Ibadov, S. 1986, ESA SP-250, 377

Ibadov, S. 1992, Astron. Zh. (USSR) 69, 737

Ibadov, S. 1996, Physical Processes in Comets and Related Objects, Cosmosinform Publ. Comp., Moscow, 181

Kaler, J. B. 1976, Astrophys. J. Suppl. Ser. 31, 517

Kostyakova, E. B. 1982, Physics of Planetary Nebulae, Nauka, Moscow, 127

Kostyakova, E. B. \& Arkhipova, V. P. 2009, Astron. Zh. (RF), 86, 1237

Kwok, S. 2000, The Origin and Evolution of PNe, CUP, Cambridge, 243

Kwok, S., Koning, N., Huang, H.-H., \& Churchwell, E. 2006, Proc. IAUS 234 Planetary Nebulae in Our Galaxy and Beyond, Cambridge Univ. Press, 2, 445

Zeldovich, Ya. B. \& Raizer, Yu. P. 1966, Physics of Shock Waves and High Temperature Hydrodynamic Phenomena, Nauka, Moscow, 686 